\title{
LA SOBREEDUCACIÓN
} y la informalidad en el mercado laboral mexicano

\begin{abstract}
Javier Martinez Morales Nayely Yolanda Balbuena Saldivar Iván Alfredo Ibarra Sánchez
\end{abstract}

Universidad Autónoma de Chihuahua

RECIBIDO: 10 DE MAYO DE 2017 ACEPTADO: 28 DE AGOSTO DE 2017

\section{RESUMEN}

T a sobreeducación, definida como el rubro de 1 profesionistas en puestos de trabajo que requieren de menor educación a la adquirida, ha sido un fenómeno que ha impactado en los últimos años en el mercado laboral mexicano. Por ello, esta investigación tiene como objetivo responder las siguientes preguntas: ¿cuál es el efecto de la sobreeducación e informalidad en el ingreso de los profesionistas?, ¿cuál es la probabilidad de estar sobreeducado, por tipo de carreras, en el mercado labo- ral de los profesionistas?, ¿cuál es la probabilidad de estar en el mercado informal, a pesar de tener una carrera profesional?, ¿cuál es la probabilidad de no estar sobreeducado? La base de datos se tomó de la Encuesta Nacional de Ocupación y Empleo (ENOE) 2016-IV trimestre. La metodología propuesta es generar una ecuación tipo minceriana y un modelo tipo probit multinomial. Los principales resultados revelan que estar en un mercado sobreeducado, reduce el ingreso en 24.6 $\%$, mientras que estar en el mercado informal lo reduce en 44.5 
$\%$. La probabilidad de estar sobreeducado en forma global, es de $33 \%$, mientras que estar en el mercado informal es de $14 \%$.

Palabras clave: sobreeducación, modelos probit, informalidad.

\section{Abstract}

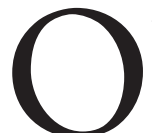

ver-education, defined as the category of professionals in jobs that require less education to the acquired, has been a phenomenon that has impacted in recent years in the Mexican labor market. Therefore this research aims to answer the following questions: What is the effect of overeducation and informality on the income of professionals? What is the probability of being over-educated, by type of careers, in the job market of professionals? What is the probability of being in the informal market despite having a professional career? What is the probability of not being over-educated? The database was taken from the National Occupation and Employment Survey (ENOE) 2016-IV quarter. The proposed methodology is to generate a mincerian equation and a multinomial probit type model. The main results were that being in an over-educated market reduces income by $24.6 \%$, while being in the informal market reduces it by $44.5 \%$. The probability of being over-educated globally is $33 \%$, while being in the informal market is $14 \%$.

Keywords: over-education, probit models, informality.

\section{INTRODUCCIÓN}

U na de las principales problemáticas de los mercados laborales en México, es el desfase que existe entre la oferta y la demanda laborales, principalmente en trabajadores con un nivel de educación profesional. Si bien la Teoría del capital humano, desarrollada por Gary Becker en 1964, establece que un mayor grado de escolaridad incrementa los niveles de ingreso de las personas posicionadas en un mercado laboral, esta no llega a explicar lo que sucede con los individuos que tienen un mismo nivel de escolaridad (profesionistas) y diferentes niveles de ingreso (Angulo, Quejada, \& Yánez, 2012). Para ello, Piore (1969; 1975), citado en Fer- nández (2010), establece que existe una estructura en el mercado dualista, el cual está compuesto por el sector primario definido por salarios altos, estabilidad laboral, oportunidades de crecimiento, etcétera, mientras que el sector secundario se caracteriza por todo lo contrario al sector primario.

Más adelante, el propio Piore subdivide al sector primario en dos mercados más: superior e inferior; en este último, se identifica básicamente a los trabajadores con especialidad técnica, mientras que en el superior se encuentran los puestos directivos y gerenciales, entre otros. A partir del enfoque estructuralista, se define que las diferencias salariales se centralizan en factores estructurales que se relacionan con "las características del puesto del trabajo, de las empresas y de los sectores industriales" (Fernández, 2010).

Hernández, Solís, \& Stefanovich (2012) elaboran un diagnóstico, para el periodo 2000-2009, sobre el mercado laboral en México; sus principales resultados muestran que hay 2.8 millones de profesionistas más durante todo el periodo, de los cuales $16 \%$ per- 
maneció inactivo; es decir; la tasa de desempleo tuvo un crecimiento promedio anual de $15 \%$. La investigación divide a los ocupados en tres grupos: en el primero están los profesionistas que tienen puestos de acuerdo con su perfil; en el segundo, se ubican los comerciantes y técnicos, y el tercero, se caracteriza por tener trabajos que pueden ser realizados independientemente de contar con una profesión o no. Los principales resultados establecen que $60 \%$ de los profesionistas estaban empleados en el primer grupo, empero hubo un incremento considerable en los otros dos grupos; los autores concluyen que este desplazamiento, se debió principalmente al excedente de oferta en el primer grupo.

Dado lo anterior, el mercado informal ha sido también una alternativa para explicar parte de esta segmentación, que está focalizado principalmente en el empleo informal y se define como las personas que no tienen ningún tipo de seguridad legal y social (Alter, 2012). De hecho, Portes, Castells y Benton (1989), citados en Samaniego (2008), determinan que las empresas

Tabla 1. Ocupaciones afines a lo que estudiaron.

\begin{tabular}{cc}
\hline Áreas & $\begin{array}{c}\text { Se ocupan en lo que estudia- } \\
\text { ron (\%) }\end{array}$ \\
\hline Educación & 91.2 \\
\hline Ciencias de la salud & 88.7 \\
\hline Ciencias físico-matemáticas & 88.6 \\
\hline Humanidades & 86.8 \\
\hline Artes & 86.1 \\
\hline $\begin{array}{c}\text { Arquitectura, urbanismo y } \\
\text { diseño }\end{array}$ & 84.2 \\
\hline Ciencias sociales & 82.1 \\
\hline Ciencias biológicas & 78.1 \\
\hline Económico-administrativas & 73.7 \\
\hline Ingenierías & 71.9
\end{tabular}

Fuente: Observatorio Laboral Mexicano con base en datos de la ENOE (2016).

afrontan demandas inestables, que les conlleva a una reestructuración que involucra procedimientos de fabricación más flexibles y eficientes, lo que permite descentralizar procesos de producción y reducir costos (principalmente de capital variable) con el objetivo de que los shocks en la demanda sean trasladados hacia el exterior de la firma.

De acuerdo con el Observatorio Laboral Mexicano (2017), con datos de la Encuesta Nacional de Ocupación y Empleo (ENOE) del tercer trimestre de 2016, el número de profesionistas ocupados ascendía a 7.8 millones de perso- nas y de estas, cinco millones se focalizaron en las áreas de ingeniería, educación y económico-administrativas. Sin embargo, la tabla 1 evidencia que no todas las personas empleadas estaban en puestos acorde con lo que estudiaron. Como se observa, las áreas económico-administrativas e ingenierías son las que menos posicionan puestos que tienen que ver con lo que estudiaron, a pesar de ser las que mayor población ocupada tienen (Observatorio Laboral Mexicano, 2017).

Con base en el planteamiento del problema, este documento tiene como objetivo analizar si se presenta un 
problema de sobreeducación e informalidad en el mercado laboral mexicano de profesionistas desde la perspectiva de las características del propio trabajador. Por lo tanto, se plantea como objetivo responder las siguientes preguntas:

a) ¿Cuál es el efecto de la sobreeducación e informalidad en el ingreso de los profesionistas?

b) ¿Cuál es la probabilidad de estar sobreeducado, por tipo de carreras, en el mercado laboral de los profesionistas?

c) ¿Cuál es la probabilidad de estar en el mercado informal, a pesar de tener una carrera profesional?

d) ¿Cuál es la probabilidad de no estar sobreeducado?

La hipótesis que se presenta en este trabajo define que la sobreeducación es una variable que tiene efectos negativos en los ingresos de las personas y que, además, la informalidad se ha vuelto un escape a este tipo de "mercados", como una alternativa de trabajo.

Para ello, este documento se divide en cinco secciones: la primera es esta introducción; en la segunda parte, se presenta la revisión de la literatura que sustenta la metodología; en la tercera, se habla sobre la obtención de información, la metodología y los modelos teóricos y prácticos; posteriormente, una cuarta sección, donde se presentan la interpretación y los resultados de los modelos econométricos; $y$, finalmente, se incluyen las conclusiones del documento.

\section{MARCo TEÓRICO}

$\mathrm{L}$ a sobreeducación se refiere al grupo de profesionistas que están ocupando puestos de trabajo con requerimientos menores a su nivel de escolaridad (McGuinness, 2006); por ejemplo, cuando un ingeniero ocupa un empleo de taxista.

Mora (2008) cita algunas características que explican por qué se presenta el fenómeno de la sobreducación, entre ellas, roce temporal del mercado laboral, información incompleta, poca o nula movilidad geográfica, movilidad laboral y efectos de señalización.

En el caso mexicano, Hernández (2004) expresa que el problema de la sobreeducación se basa, en parte, en el bajo crecimiento económico del país.

De acuerdo con el estudio de Gobernado (2007), existen tres formas de medir la sobreeducación: la primera es la objetiva, que se centraliza en ocupar medidas oficiales que se basan en el grado escolar focalizado en el tipo de empleo; la segunda es el método empírico, que consiste en calcular el valor medio de los niveles educativos de los puestos laborales, donde aquellos empleos con educación por encima de la media son sobreeducados; la tercera es la subjetiva, que se basa en una pregunta directa al empleado, específicamente: ¿cree usted que sus conocimientos y habilidades son equiparables a su puesto laboral?

Verdugo y Turner (1989) muestran, con datos del Censo de Estados Unidos de 1980, que la sobreeducación genera $13 \%$ menos de ingreso, mientras que la subeducación reduce en $10 \%$ el ingreso comparado con los adecuadamente educados para el puesto. Su metodología se basa en la ecuación minceriana y consideran variables como edad, sexo, experiencia, sector, ocu- 
pación, región, horas de trabajo y estado civil.

Chevalier (2003) considera una división de la sobreeducación en los trabajadores por sus tipos de habilidades (ocupa una muestra de 1985 y 1990 de Reino Unido), tomando en cuenta dos tipos de sobreeducación: los que aparentemente están sobreeducados (personas que no tienen profesión) y los que genuinamente sí están sobreeducados (individuos con grado profesional o mayor). La sobreeducación aparente reduce el ingreso entre 5 y $11 \%$, mientras que la genuinamente lo reduce entre 22 a $26 \%$.

De la Garza y Villezca (2006), con datos de la Encuesta Nacional de Empleo Urbano (ENEU) 2002, evidencian que una persona sobreeducada gana $19 \%$ menos de ingreso que una que tiene un puesto laboral acorde con sus estudios, considerando las características del profesionista, como edad, sexo, horas trabajadas, maestría, doctorado, empresa (pública o privada) y carrera profesional.

De Vries y Navarro (2011) evidencian, a través de una muestra de 4260 cuestionarios aplicados en 2007 y 2008 a egresados de licenciatura, tanto de universidades públicas como privadas, que cerca de $15 \%$ ocupaban un puesto directivo, mientras que $45 \%$ de profesionistas científicos ocupaban puestos de empleados, técnicos u otros; sin embargo, más de $93 \%$ sentían que era necesario tener un grado de licenciatura o mayor para el puesto requerido. El $33 \%$ respondieron que su área estaba de acuerdo con lo que requería el trabajo, mientras que el resto dijeron que no era así necesariamente.

Burgos y López (2011) aplicaron una encuesta a egresados de la Universidad de Sonora y sus empleadores, para medir el efecto de la sobreeducación; ocuparon el método subjetivo y sus principales resultados demuestran que $11.1 \%$ de los encuestados tuvieron una percepción de estar sobreeducados. Por otro lado, sus estimaciones determinaron que una mayor capacitación, contratación indeterminada, maestrías o doctorados y ser hombre, permiten tener un mayor salario, mientras que estar sobreeducado reduce el ingreso salarial cerca de $19.38 \%$.
Por otro lado, desde la medición de la probabilidad de estar sobreeducado o no existen estudios como el de Mora (2008), quien determinó que ser mujer, tener experiencia y trabajar en una empresa mediana o grande, reducen la probabilidad de estar sobreeducado, mientras que estar en una microempresa, tener movilidad entre áreas de desempeño y trabajar en el sector primario, aumentan la probabilidad de estar sobreeducado. El trabajo de Mora implica no solo la sobreeducación, sino también la subeducación.

Domínguez (2006), basado en Thurow (1972), resume que existe una teoría sobre la informalidad, que consiste en que los puestos de trabajo se forman en función de una cola, los cuales se justifican con las características de las personas, tales como edad, sexo y experiencia, entre otras. Esta cola ordena a los trabajadores como los "mejores" y los "peores"; sin embargo, cuando el trabajador no queda en esta división, debido a la escasez de trabajo, lo expulsa a la informalidad. Con base en el método subjetivo, sus resultados muestran que una mayor so- 
breeducación conlleva a ganar $20 \%$ menos que quienes no están sobreeducados. Por otro lado, el mismo autor calcula, a través de un modelo binomial, el efecto de la sobreeducación sobre la probabilidad de estar en el mercado formal y su principal resultado determina que la sobreeducación reduce la probabilidad de estar en el mercado formal.

En el mismo sentido, Herrera-Idárraga, López-Bazo y Motellón (2013), con microdatos de Colombia, estiman que estar en un mercado formal reduce la probabilidad de estar sobreeducado en $2.5 \%$ con respecto a estar en el mercado informal.

Sin embargo, Alcaraz, Chiquiar y Salcedo (2015) definen que la segmentación del mercado laboral permite saber si el trabajador puede o no pasar del mercado informal al formal, debido a algunas barreras, pero, por otro lado, también existe la posibilidad de que el trabajador informal se encuentre en este mercado, porque él así lo decidió. Sus resultados muestran que entre 10 y $20 \%$, los trabajadores optaron por pasar del mercado informal al formal. Sin embargo, también se encontró evidencia de que hay trabajadores que se autoseleccionan en el mercado informal.

\section{Metodología}

T a base de datos se obtuvo de la ENOE 2016 (cuarto trimestre). Se considerará únicamente a la población ocupada que declaró estar como trabajadores subordinados o remunerados; se descarta otro tipo de población ocupada y únicamente se toma en cuenta a los profesionistas con carrera terminada. Se estratifica a las empresas como micro, pequeñas, medianas, grandes y de gobierno; es importante mencionar que se tomó en cuenta solo a los trabajadores que expresaron el tamaño de la empresa. Para medir la variable sobreeducación, se propone el método objetivo, ya que la ENOE no cuenta con preguntas tan específicas para ocupar el método subjetivo. Para el caso de la informalidad, también se considera el cálculo emitido por la ENOE (2014) y que en la propia base de datos ya está clasificada.

Las carreras se concentrarán en los siguientes grupos:
Educación (ciencias de la educación y formación docente); Artes y humanidades; Ciencias sociales (ciencias sociales, estudios del comportamiento y ciencias de la información); Negocios y administración; Derecho; Ciencias naturales, exactas y de la computación; Ingeniería y manufactura (ingeniería industrial, mecánica, electrónica y tecnología, manufactura y procesos); Arquitectura y construcción; Ciencias de la salud; y Servicios.

Se considera la población con ingreso cero, porque representa cerca de $32 \%$ del total de las observaciones. Finalmente, se tendrá una muestra de 22173 observaciones de una población, de acuerdo con el factor de expansión de 6021114 profesionistas del país.

Para el análisis y estimación de las preguntas de investigación, se trabaja con dos modelos econométricos: el primero estima el efecto de la sobreeducación e informalidad en el ingreso de las personas. Para su estimación, se propone el modelo de De la Garza y Villezca (2006) y sustituyendo las variables empresa pública por tamaño de la empresa y ca- 
sado por soltero, se estimó el siguiente modelo desde el método de mínimos cuadrados generalizados con base en Rojas, Angulo y Velázquez (2000).

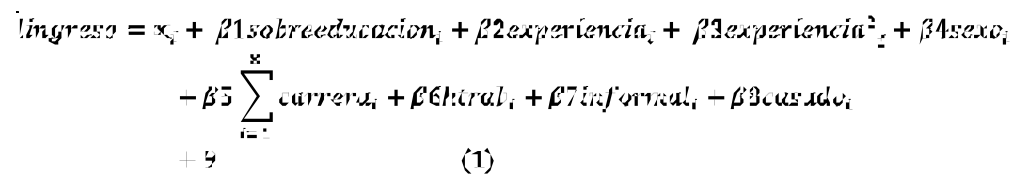
Donde:

- Ingreso = logaritmo del ingreso de los trabajadores;

- Sobreeducación $=1$, si está sobreeducado; y 0 , si no lo está;

- Experiencia: la variable experiencia se generó con base en el modelo propuesto por Huesca (2004,) calculada como Experiencia $=$ Edad-Escolaridad acumulada -6 años de ocio. Dada la alta correlación que presentan las variables edad y experiencia, se dejó fuera la variable edad del modelo;

- Sexo $=0$, si es mujer; y 1 , si es hombre;

- Carreras: Educación (ciencias de la educación y formación docente); Artes y humanidades; Ciencias sociales (ciencias sociales, estudios del comportamiento y ciencias de la información); Negocios y administración; Derecho; Ciencias naturales, exactas y de la computación; Ingeniería y manufactura (ingeniería industrial, mecánica, electrónica y tecnología, manufactura y procesos); Arquitectura y construcción; Ciencias de la salud; y Servicios;

- htrab = horas trabajadas;

- Informal $=0$, si el profesionista está en el mercado formal; y 1 , si se encuentra en el mercado informal;

- Casado $=1$, si el profesionista está casado; y 0 , para cualquier otra relación.

Para medir la variable sobreeducación, se siguió el método objetivo, dado que la ENOE no cuenta con preguntas específicas para utilizar el método subjetivo. La variable dependiente se generó en función de tres grupos; primer grupo: educado correctamente y formal; segundo grupo: sobreeducado y formal; y tercer grupo: informal (sin importar si dentro de este mercado estuviera 
sobreeducado o no). Para estimar la probabilidad de la sobreeducación, se ocupó un modelo tipo probit multinomial:

$$
s^{*}=\boldsymbol{\beta}^{\prime} \mathbf{z}+\xi
$$

Donde:

$\boldsymbol{\beta}^{\prime}=$ vector de parámetros;

$z=$ variables independientes;

$\boldsymbol{\xi}=$ residuo que sigue una distribución normal en las observaciones;

$s^{*}=$ variable no observable.

Sin embargo, lo que sí se observa:

$S_{1}=1 \mid$ correctamente educado y formal,$\quad$ si $s^{*} \leq 0$

$S_{2}=2[$ Sobre educado y formal $]$, si $0<s^{*} \leq \sigma_{1}$

$S_{3}=3 \mid$ Informal,$\quad$ si $\sigma_{1}<s^{*} \leq \sigma_{2}$.

Dada la distribución del $\boldsymbol{\zeta \zeta}$, entonces se obtienen las siguientes probabilidades (Greene, 1990, p. 703):

$\operatorname{Prob}(s=1)=\delta\left(-\boldsymbol{\beta}^{\prime} \mathbf{z}\right)$

$\operatorname{Prob}(s=2)=\delta\left(\sigma_{1}-\boldsymbol{\beta}^{\prime} \mathbf{z}\right)-\delta\left(-\boldsymbol{\beta}^{\prime} \mathbf{z}\right)$

$\operatorname{Prob}(s=3)=\delta\left(\sigma_{2}-\boldsymbol{\beta}^{\prime} \mathbf{z}\right)-\delta\left(\sigma_{1}-\boldsymbol{\beta}^{\prime} \mathbf{z}\right)$

Donde:

$\delta=$ función de distribución normal estándar.

Dada la información que se tiene de la ENOE y los procedimientos anteriores, se estima la siguiente función de sobreeducación:

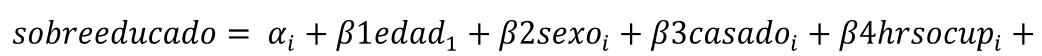
$\beta 5 \sum_{i=1}^{9}$ carrera $_{i}+\varepsilon_{i}$ 
En la tabla 2 se presenta el resumen de las variables a trabajar durante todo el documento. Las variables cuantitativas representan promedios, mientras que las cualitativas muestran la participación de cada grupo.

Tabla 2. Resumen de variables

\begin{tabular}{ccccc}
\hline Variable & Grupo 1 & Grupo 2 & Grupo 3 & Total \\
\hline Edad & 39 & 37 & 35 & 37 \\
\hline Ingreso & 7502.2 & 6996.7 & 4677.1 & 6392 \\
\hline Horas trabajadas & 37.6 & 44.5 & 38.6 & 40 \\
\hline Experiencia & 16.3 & 14.6 & 12.5 & 15 \\
\hline Hombres & 48.25 & 35.87 & 16 & $100 \%$ \\
\hline Casados & 55.62 & 32.62 & 11.76 & $100 \%$ \\
\hline Educación & 77.49 & 12.15 & 10.35 & $100 \%$ \\
\hline Artes y Humanidades & 51.62 & 22.1 & 26.28 & $100 \%$ \\
\hline Ciencias sociales & 47.42 & 33.52 & 19.06 & $100 \%$ \\
\hline $\begin{array}{c}\text { Negocios y Adminis- } \\
\text { tración }\end{array}$ & 41.24 & 43.99 & 14.77 & $100 \%$ \\
\hline Derecho & 42.17 & 36.75 & 21.08 & $100 \%$ \\
\hline $\begin{array}{c}\text { Ciencias naturales, } \\
\text { exactas y computación }\end{array}$ & 50.45 & 33.91 & 15.64 & $100 \%$ \\
\hline Ingeniería & 42.57 & 45.73 & 11.69 & $100 \%$ \\
\hline Arquitectura & 45.58 & 34.37 & 20.05 & $100 \%$ \\
\hline $\begin{array}{c}\text { Agronomía y Veteri- } \\
\text { naria }\end{array}$ & 37.97 & 39.66 & 22.36 & $100 \%$ \\
\hline $\begin{array}{c}\text { Ciencias de la salud } \\
\text { Servicios }\end{array}$ & 71.23 & 10.47 & 18.3 & $100 \%$ \\
\hline & 36 & 44.8 & 19.2 & $100 \%$ \\
\hline & & &
\end{tabular}

Fuente: elaboración propia con base en datos de la ENOE (2016). 
Figura 1. Ingresos por carrera y grupo.

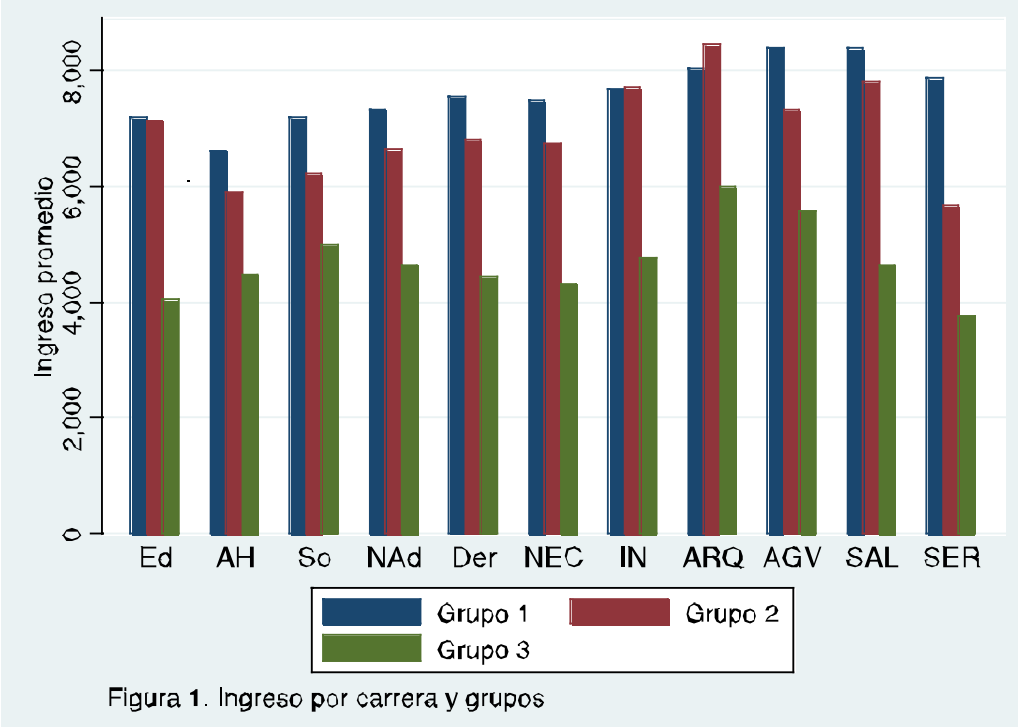

Fuente: elaboración propia.

Figura 2. Ingresos por sexo y grupo.

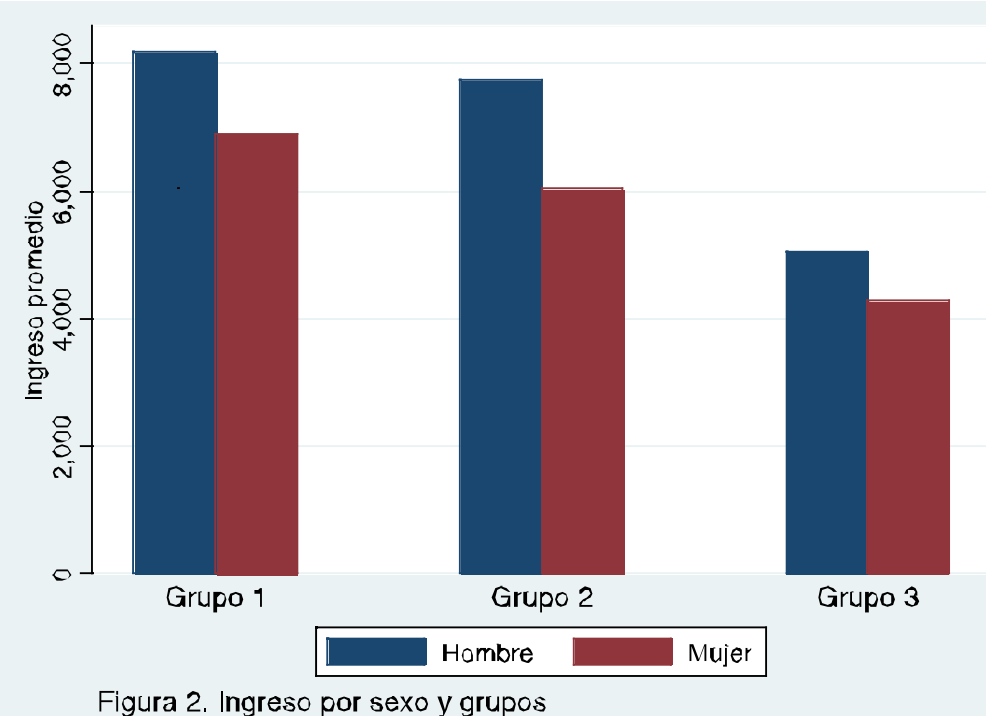

Fuente: elaboración propia.
Análisis E

INTERPRETACIÓN DE RESULTADOS

T a figura 1 presenta el ingreso promedio por tipo de carrera y tipo de grupo al que pertenecen las personas encuestadas. Como puede observarse, en todos los casos, trabajar en la informalidad conlleva a percibir menores ingresos, sin importar el tipo de carrera, mientras que en las carreras de Educación e ingenierías, los ingresos promedio de los grupos 1 y 2 son muy similares. Por otro lado, la carrera de Arquitectura tiene mayores ingresos en el grupo 2 que en el grupo 1.

La figura 2 muestra las diferencias salariales por grupo y sexo. En cualquiera de los tres grupos, en promedio, los hombres ganan más que las mujeres. Esto es un claro ejemplo de que existe cierta discriminación salarial, ya que los tres grupos están integrados por profesionistas con carrera terminada.

La tabla 3 presenta los resultados del primer modelo econométrico, esto es, la estimación del ingreso en función de las variables antes mencionadas. 
Tabla 3. Resultados del primer modelo econométrico.

\begin{tabular}{ccc}
\hline Ingreso & Coeficiente & Error est. \\
\hline Sobreeduc. & $-0.2464702^{* * *}$ & 0.0135585 \\
\hline Exper. & $0.020724^{* * *}$ & 0.0016032 \\
\hline Exper2 & $-0.0002848^{* * *}$ & 0.0000432 \\
\hline Sexo & $-0.1305058^{* * *}$ & 0.0098989 \\
\hline Educación & -0.0018268 & 0.0257282 \\
\hline Sociales & 0.053926 & 0.0291949 \\
\hline Neg. & & \\
& $0.0661748^{* *}$ & 0.0260753 \\
\hline yadmón. & & \\
\hline Derecho & $0.1094261^{* * *}$ & 0.0292159 \\
\hline Naturales & 0.0164353 & 0.0310179 \\
\hline Ingeniería & $0.1499515^{* * *}$ & 0.0279703 \\
\hline Arquitectura & $0.2056406^{* * *}$ & 0.0331766 \\
\hline Agronomía & -0.0212123 & 0.0361168 \\
\hline Salud & $0.1510902^{* * *}$ & 0.0285288 \\
\hline Ser & 0.0751892 & 0.0702613 \\
\hline Horas ocup. & $0.0049602^{* * *}$ & 0.0004067 \\
\hline Mercado & $-0.4455554^{* * *}$ & 0.0145836 \\
\hline Casado & $0.0806479^{* * *}$ & 0.0096957 \\
\hline Constante & $8.877037^{* * *}$ & 0.0352553 \\
\hline
\end{tabular}

Nota: ${ }^{*} p<.05 ;{ }^{* *} p<.01 ;{ }^{* * *} p<.001$

Fuente: elaboración propia.
En primer lugar, observamos que la sobreeducación reduce el ingreso de las personas en $25 \%$, aproximadamente, manteniendo todas las demás variables constantes. Aplicando la fórmula de la $\mathrm{U}$ invertida para la experiencia, se determina que el valor umbral es de treinta y siete años, así como que el hecho de ser mujer reduce el ingreso en $13.99 \%$. Se observa que la carrera que mayor impacto tiene sobre el ingreso, es Arquitectura, mientras que estar en el mercado informal reduce el ingreso cerca de $44 \%$. A mayores horas trabajadas aumenta el ingreso, es decir, si se incrementa una hora de trabajo, el ingreso aumenta en $0.49 \%$, mientras estar casado incrementa el ingreso en $8.32 \%$. La carrera de artes y humanidades fue la variable base.

Para el segundo modelo, la tabla 4 exhibe los resultados econométricos. 
Tabla 4. Efectos marginales después del modelo probit.

\begin{tabular}{|c|c|c|c|}
\hline Probabilidad de $Y$ & Grupo 1 & Grupo 2 & Grupo 3 \\
\hline & $53 \%$ & $33 \%$ & $14 \%$ \\
\hline Variable & $d y / d x$ & $d y / d x$ & $d y / d x$ \\
\hline Edad & $\begin{array}{c}0.0046524 \\
\left(0.00034^{* *}\right)\end{array}$ & $\begin{array}{c}-.002015 \\
\left(0.00015^{* *}\right)\end{array}$ & $\begin{array}{l}-0.0026374 \\
\left(0.00019^{* *}\right)\end{array}$ \\
\hline Sexo & $\begin{array}{c}0.0302633 \\
\left(0.00687^{* *}\right) \\
\end{array}$ & $\begin{array}{c}.0131074 \\
\left(0.00298^{* *}\right) \\
\end{array}$ & $\begin{array}{c}-0.0171559 \\
\left(0.0039^{* *}\right) \\
\end{array}$ \\
\hline Educación & $\begin{array}{c}0.2270458 \\
\left(0.01132^{* *}\right) \\
\end{array}$ & $\begin{array}{c}-.1184081 \\
\left(0.00716^{* *}\right) \\
\end{array}$ & $\begin{array}{l}-0.1086376 \\
\left(0.00451^{* *}\right)\end{array}$ \\
\hline Artes y human. & $\begin{array}{c}-0.0366963 \\
(0.02054) \\
\end{array}$ & $\begin{array}{l}.0149751 \\
(0.00784) \\
\end{array}$ & $\begin{array}{c}0.0217212 \\
(0.01271) \\
\end{array}$ \\
\hline Ciencias sociales & $\begin{array}{c}-0.0248594 \\
(0.013659) \\
\end{array}$ & $\begin{array}{c}.0103907 \\
\left(0.00549^{*}\right) \\
\end{array}$ & $\begin{array}{c}0.0144686 \\
(0.00816) \\
\end{array}$ \\
\hline Neg. y Admón. & $\begin{array}{l}-0.0363956 \\
\left(0.00936^{* *}\right)\end{array}$ & $\begin{array}{c}.0152959 \\
\left(0.00382^{*}\right) \\
\end{array}$ & $\begin{array}{c}0.0210997 \\
\left(0.00556^{* *}\right)\end{array}$ \\
\hline Derecho & $\begin{array}{l}-0.0653083 \\
\left(0.01249^{* *}\right) \\
\end{array}$ & $\begin{array}{c}.0256739 \\
\left(0.0044^{* *}\right) \\
\end{array}$ & $\begin{array}{c}0.0396343 \\
\left(0.00813^{* *}\right)\end{array}$ \\
\hline Ciencias naturales & $\begin{array}{c}0.0106239 \\
(0.01508) \\
\end{array}$ & $\begin{array}{r}-.0046722 \\
(0.00673) \\
\end{array}$ & $\begin{array}{c}-0.0059517 \\
(0.00834) \\
\end{array}$ \\
\hline Arquitectura & $\begin{array}{c}-0.0437443 \\
\left(0.0158^{*}\right) \\
\end{array}$ & $\begin{array}{c}.0176792 \\
\left(0.00591^{*}\right) \\
\end{array}$ & $\begin{array}{l}0.0260652 \\
\left(0.00991^{*}\right) \\
\end{array}$ \\
\hline Agronomía & $\begin{array}{c}-0.132737 \\
\left(0.02073^{* *}\right) \\
\end{array}$ & $\begin{array}{c}.0440755 \\
\left(0.00461^{* *}\right)\end{array}$ & $\begin{array}{c}0.0886615 \\
\left(0.01624^{* *}\right)\end{array}$ \\
\hline Ciencias de la salud & $\begin{array}{c}0.134643 \\
\left(0.01449^{* *}\right) \\
\end{array}$ & $\begin{array}{c}-.0679182 \\
\left(0.00845^{* *}\right) \\
\end{array}$ & $\begin{array}{l}-0.0667247 \\
\left(0.00614^{* *}\right)\end{array}$ \\
\hline Servicios & $\begin{array}{c}-0.0999351 \\
\left(0.03696^{*}\right) \\
\end{array}$ & $\begin{array}{c}.0356522 \\
\left(0.01013^{* *}\right)\end{array}$ & $\begin{array}{l}0.0642829 \\
\left(0.02686^{*}\right) \\
\end{array}$ \\
\hline Horas ocupadas & $\begin{array}{l}-0.0011119 \\
\left(0.00026^{* *}\right) \\
\end{array}$ & $\begin{array}{c}.0004816 \\
\left(0.00012^{* *}\right) \\
\end{array}$ & $\begin{array}{l}0.0006303 \\
\left.0.00015^{* *}\right) \\
\end{array}$ \\
\hline Casado & $\begin{array}{c}0.0523993 \\
\left(0.00674^{* *}\right)\end{array}$ & $\begin{array}{c}-.0227041 \\
\left(0.00293^{* *}\right)\end{array}$ & $\begin{array}{c}-0.0296951 \\
\left(0.00385^{* *}\right)\end{array}$ \\
\hline
\end{tabular}

Nota: ${ }^{*} p<.05 ;{ }^{* *} p<.01 ;{ }^{* * *} p<.001$

Fuente: elaboración propia.
Se observa que hay una probabilidad de $53 \%$ de estar en el grupo $1 ; 33 \%$, en el grupo 2; y $14 \%$, en el grupo 3. Es decir, la informalidad y la sobreeducación absorben una probabilidad de $47 \%$. A medida que la edad aumenta, la probabilidad se incrementa en $0.46 \%$ de estar el grupo 1 $y$, por ende, reduce la probabilidad de estar en los otros dos grupos. Ser hombre aumenta la probabilidad de estar en el grupo 1 en $3.0 \%$. Estudiar las carreras de Educación y de ciencias de la salud aumenta la probabilidad de estar en el grupo 1 con respecto al área de ingeniería. Las carreras con mayor probabilidad de estar sobreeducadas son Negocios y Administración. Finalmente, las carreras de Agronomía y Servicios son las que más alta probabilidad tienen de estar en el grupo 3, es decir, en el mercado informal.

\section{Conclusiones}<smiles>[PbH2]</smiles>
l estudio llegó a responder las preguntas objetivo del planteamiento del problema y a fundamentar la hipótesis planteada. La sobreeducación reduce el nivel de ingresos de las personas; sin 
embargo, el efecto de la informalidad reduce aún más el ingreso de los profesionistas. No cabe duda de que los mercados informales empiezan a ser una alternativa de los empujes de los grupos "mejores" a los "peores".

El fenómeno de la sobreeducación debe ser estudiado con precaución, sobre todo en su medición, porque desde esa perspectiva los resultados pueden variar de una investigación a otra.

A pesar de que la probabilidad de estar en un mercado informal aún es baja, no deja de ser relevante terminar en él. Esto puede derivar a que los mercados informales se vuelvan un escape de los problemas de oferta y demanda de los mercados laborales para profesionistas.

Finalmente, se puede decir que tanto el mercado informal como la sobreeducación no son fenómenos aislados, sino que, al parecer, se comportan como sustitutos o complementarios a la vez, lo que implica una seriedad grave para la educación profesional.

\section{REFERENCIAS}

\section{BIBLIOGRÁFICAS}

Alcaraz, C., Chiquiar, D., \& Salcedo, A. (2015). Informality and Segmentation in the Mexican Labor Market. Working Papers, 20152025. México: Banco de México.

Alter Chen, M. (2012). La economía informal: definiciones, teorías y políticas. WIEGO Mujeres en empleo informal: globalizando y organizando. Documento de trabajo de WIEGO, 1. Recuperado de http://www.inclusivecities.org/wp-content/uploads/2013/06/ Chen_WIEGO_WP1_ es.pdf

Angulo Pico, G. M., Quejada Pérez, R., \& Yánez Contreras, M. (2012). Educación, mercado de trabajo y satisfacción laboral: el problema de las teorías del capital humano y señalización de mercado. Revista de la Educación Superior, XLI(163), 51-66. Recuperado de http://resu.anuies. $\mathrm{mx} /$ archives/revistas/Revista163_S1A2ES.pdf

Burgos Flores, B., \& López Montes, K. (2011). Efectos de la sobreeducación y el desfase de conocimientos sobre los salarios y la búsqueda de trabajo de profesionistas. Resultados de un estudio basado en opiniones y percepciones de egresados universitarios y empleadores. Perfiles Educativos, 33(134), 34-51.

Chevalier, A. (2003). Measuring Over-education. Economica, 70(3), 509-531.

De la Garza Garza, Ó. J., \& Villezca Becerra, P. A. (2006). Efecto de la sobreeducación en el ingreso de las personas con estudios de nivel superior en México. Ensayos, $X X V(2), 21-$ 42. Recuperado de http:// eprints.uanl.mx/7544/

De Vries, W., \& Navarro, Y. (2011). ¿Profesionistas del futuro o futuros taxistas? Los egresados universitarios y el mercado laboral en México. Revista Iberoamericana de Educación Superior (RIES). México, II(4), 3-27. Recuperado de http://ries.universia.net/ index.php/ries/article/ view/71

Domínguez, J. (2006). Sobreeducación en el mercado laboral urbano de Colombia para el año 2006. Do- 
cumento de trabajo, 120. Cali, Colombia: Universidad del Valle, Cidse. Recuperado de http:// bibliotecavirtual.clacso. org.ar/Colombia/cidseunivalle/20101014100850/ DOCTRAB120.pdf

Encuesta Nacional de Ocupación y Empleo (ENOE) (2016). Primer trimestre. Instituto Nacional de Estadística y Geografía. Recuperado de http://www. beta.inegi.org. $\mathrm{mx} /$ proyectos/enchogares/regulares/ enoe/

(2014). La informalidad laboral. Marco conceptual y metodológico.

Fernández Huerga, E. (2010). La Teoría de la segmentación del mercado de trabajo: enfoques, situación actual y perspectivas del futuro. Investigación Económica, 69(273), 115-150. Recuperado de http://www.scielo.org.mx/ scielo.

php?script=sci_arttext\&pid $=$ S018516672010000300004

Gobernado Arribas, R. (2007). La sobreeducación en España: estudio descriptivo y revisión crítica del concepto. Papers: Revista de Sociología, 86, 11-31. Recuperado de http://papers.uab.cat/article/view/ v86-gobernado

Greene, W. H. (1990). Econometric Analysis. New York: Macmillan Publishing Company.

Hernández Laos, E. (2004). Panorama del mercado laboral de profesionistas en México. Economía UNAM, 1(2), 98-109.

Hernández Laos, E., Solís Rosales, R., \& Stefanovich Henchoz, A. F. (2012). Mercado laboral de profesionistas en México. Diagnóstico 2000-2009 y prospectiva 2010-2020. Informe final (Col. Documentos). México: ANUIES.

Huesca Reynoso, L. (2004). La rentabilidad de la escolaridad en los hogares asalariados de México durante 1984-2000. Problemas del Desarrollo. Revista Latinoamericana de Economía, 35(138). Recuperado de http://www.ejournal. unam.mx/pde/pde138/ PDE13807.pdf

Herrera-Idárraga, P., LópezBazo, E., \& Motellón, E. (2013). Informality and Over-education in the La- bor Market of a Developing Country. Working Paper, 2013/05, 1-34. Barcelona, Spain: Research Institute of Applied Economics.

McGuinness, S. (2006). Over-education in the Labour Market. Journal of Economic Surveys, 20(3), 387-418. Recuperado de http://onlinelibrary.wiley. com/doi/10.1111/j.09500804.2006.00284.x/full

Mora, J. J. (2008). Sobreeducación en el mercado laboral colombiano. Revista de Economía Institucional, 10(19), segundo semestre, 293-309. Recuperado de https://www.economiainstitucional.com/pdf/ No19/jmora19.pdf

Rojas, M., Angulo, H., \& Velázquez I. (2000). Rentabilidad de la inversión en capital humano en México. Economía Mexicana. Nueva Época, $I X(2)$, segundo semestre, 113-142. Recuperado de http://www. economiamexicana.cide. edu/num anteriores/IX2/01_MARIANO_ROJAS_113-142.pdf

Samaniego, N. (2008). El crecimiento explosivo de la economía informal. Eco- 
nomía UNAM, 5(13), 3041. Recuperado de http:// www.revistas.unam.mx/ index.php/ecu/article/ view/2935/2495

Secretaría del Trabajo y Prevención Social (sTPS). Observatorio Laboral. Recuperado de http://www. observatoriolaboral.gob. $\mathrm{mx} / \mathrm{swb} / \mathrm{es} / \mathrm{ola} /$ tendencias_del_empleo_profesional

Verdugo, R. R., \& Turner Verdugo, N. (1989). The Impact of Surplus Schooling on Earnings: Some Aditional Findings. The Journal of Human Resources, 24(4), 629-643. Recuperado de http://www.jstor.org/stable/145998 